|| Print ISSN: 2589-7837 || Online ISSN: 2581-3935 ||

International Journal of Medical Science and Diagnosis Research (IJMSDR)

Available Online at www.ijmsdr.com

NLM (National Library of Medicine ID: 101738824)

Original Research Article

Volume 5, Issue 11; November:2021; Page No. 01-06

\title{
Minimising Radiation Dose in Computerised Tomography in the Investigation of Renal Stone Disease: A Teaching Hospital Audit
}

\section{Andrew Refalo}

\author{
MBChB (Hons), BSc (Hons), The University of Sheffield
}

\author{
Conflicts of Interest: Nil \\ Corresponding author: Andrew Refalo \\ DOI: https://doi.org/10.32553/ijmsdr.v5i11.869
}

\begin{abstract}
:
Background: CTKUB is a commonly performed scan as the gold standard investigation for renal stone disease. However, CT exposes a patient to ionising radiation. The radiation delivered to the patient increases in importance given the young demographic and often need for interval imaging. To minimise exposure, low dose scanning techniques are employed, and image field is reduced to only scan relevant anatomy. A common starting point for CT KUB is often the T10 vertebra however many scans are performed starting at higher levels exposing the patient to unnecessary radiation.

Methods: A list of 100 patients who underwent CTKUB for renal stone disease at a teaching hospital was created. Following exclusion of duplicates and application of exclusion criteria 51 scans were included. Each scan was individually analysed to assess vertebral level of upper limit of kidney and vertebral level at which the CTKUB scan was started. Percentage overshoot was calculated as number of axial slices cranial to the upper aspect of the kidneys.

Results: $22 \%$ of scans included in the study were started proximal to T10, these scans had a mean percentage overshoot of $19.6 \%$ exposing the patient to excess radiation. Of the CT KUB scans started at T10 or below mean percentage overshoot was improved to $8.6 \%$. At the level of T10 all analysed scans would avoid undershooting.

Conclusions: Implementation of a CTKUB imaging protocol which starts at T10 will minimise ionising radiation exposure whilst allowing visualisation of the complete renal tract.
\end{abstract}

Keywords: CT KUB, Radiation safety, Minimise radiation

\section{Introduction:}

Renal calculi are a common condition with an estimated prevalence of $10 \%$ in males and $7 \%$ in females ${ }^{1,2}$. Patients often present acutely with severe pain which may radiate from flank(loin) down to the groin or genitalia of the individual ${ }^{3}$. Computerised tomography of the renal tract (kidney, ureters and bladder) is the gold standard investigation when investigating acute renal colic in an adult ${ }^{4,5}$. However, CT KUB involves ionising radiation. As these scans become more common, with patients sometimes requiring repeat interval scanning, it is important to minimise the potential harm of the investigation.

A method of reducing ionising radiation exposure to the patient is by utilising a low-dose (LD) technique. Low dose CT KUB protocols have been shown to reduce the total dose to the patient by 58 $88 \%^{6-8}$. This helps minimise radiation exposure whilst maintaining the diagnostic capabilities of the scan ${ }^{9}$. Another useful method of ensuring minimal exposure is to ensure only relevant anatomy is scanned. By limiting cross sectional imaging to only relevant anatomy required to answer the clinical question ionising radiation exposure is reduced. For CT KUB in the assessment of renal colic, this involves starting scanning from the upper pole of the highest kidney. Anatomy imaged cranial to the upper pole of the highest kidney provides no diagnostic benefit in the assessment of renal stone disease. Anatomical landmarks to start a CT KUB are often based on vertebral body ${ }^{10-12}$. 
The kidneys are a retroperitoneal organ, commonly found between the transverse processes of the thoracic vertebrae 12 (T12) cranially to lumbar vertebrae 3 (L3) caudally ${ }^{13}$. Hence, numerous scanning protocols suggest $\mathrm{T} 10$ as a starting point for $\mathrm{CT} \mathrm{KUB}^{11,14}$. However, despite this advice, many scans start above the level of $\mathrm{T} 10$, unnecessarily increasing ionising radiation exposure. Our study aims to audit CT KUB scans performed at a teaching hospital to assess upper pole kidney vertebral level and percentage overshoot proximal to this. Based on the results we aim to make recommendations to minimise radiation exposure in CTKUB.

\section{Methods}

\section{Study Participants/ Recruitment:}

This retrospective study was conducted at a teaching hospital. A worklist of the last one hundred CTKUB scans performed at the trust was obtained using PACS software. A further filter was placed onto the list looking for mention of 'renal colic' or 'renal stone' or 'stone' or 'calculi' in the $\mathrm{CT}$ request form. The following inclusion and exclusion criteria were then applied:

Inclusion: 1) CTKUB for renal stone disease 2) Age $>18$ years of age

Exclusion: 1) Duplicates, for patients who underwent serial imaging 2) CT scans which included protocol other than that for CTKUB 3) CT scans which involved injection of contrast 4) CT scans which were terminated without completion due to patient factors.

Patients over the age of 18 undergoing CTKUB for the assessment of query renal stone disease were included provided they met the inclusion criteria.

\section{Data Collection Procedure:}

The obtained PACS list, including patients from the last 12 months who had a CTKUB for investigation of renal colic was exported to excel. Excel data included anonymised patient details, time and date of CT KUB and CT scan request information. The list of patients was then manually reviewed by the author, excluding duplicates and scans which failed to meet inclusion criteria. Using anonymised patient ID codes, the imaging of each CTKUB was individually reviewed by the author.

On review of each scan, the following information was obtained: vertebral level at which the scan started; vertebral level at which the upper pole of the kidneys started; total cross-sectional slices in the axial plane; number of axial cross-sectional slices above the level of the upper pole of the kidney. The vertebral level was obtained on imaging by counting from L5 upwards.

Data processing and analysis:

The vertebral level at which the scan was started was subtracted by the vertebral level at which the upper aspect of the kidney started individually for each scan. To further assess scan overshoot, overshoot percent of axial slices proximal to the upper pole of the kidneys was calculated using the following formula:

$$
\text { Percentage overshoot }=\frac{\text { Number of axial CT slices } \text { cranial to upper pole kidneys }}{\text { Total number of axial slices }} \times 100
$$

Calculated overshoot percentage was calculated as mean for the whole data set as well as a mean for scans starting at each vertebral level.

\section{Results}

Study participants

Initial patient worklist included $100 \mathrm{CT}$ KUBs performed at the trust requested for assessment of renal colic. Following removal of duplicates and scans which were incorrectly detected on the list 84 CT KUB's remained. Inclusion and exclusion criteria were applied; following which 51 scans were included into the final study. Exclusion reasons included: Scans which included CTKUB as part of the scan however scanned further anatomy; CT scans which involved injection of contract and scans which were incomplete, or images not uploaded onto the system.

\section{CT KUB imaging}

The vertebral level at which the most proximal aspect of the kidneys started ranged from T10 to $\mathrm{L} 1$ with $4 \%$ at $\mathrm{T} 10,16 \%$ at $\mathrm{T} 11,51 \%$ at $\mathrm{T} 12$ and 
$28 \%$ at L1. None of the scans reviewed failed to image the entirety of the kidney.

The vertebral level at which the CTKUB was started ranged from $\mathrm{T} 7$ to $\mathrm{L} 1$, with $2 \%$ at $\mathrm{T} 7,8 \%$ at $\mathrm{T} 8,12 \%$ at $\mathrm{T} 9,24 \%$ at $\mathrm{T} 10,24 \%$ at $\mathrm{T} 11,24 \%$ at $\mathrm{T} 12$ and $4 \%$ at L1. Of all the scans included $22 \%$ of scans were started cranial to T10. Figure 1 illustrates the number of scans which started at each vertebral level and at which vertebral level the upper pole of the kidney was located.

Of all included scans the average overshoot in terms of percentage of axial slices obtained cranial to the upper aspect of the kidney was $13.3 \%$.
Individual percentage overshoot calculated for scans at each vertebral body ranged from $23.1 \%$ (for scans which started at T7) to $0.36 \%$ (for scans which started at L1) (Table 1). The lower the vertebral level at which the scan started the less overshoot expressed as percentage of excess axial slices (table 1). The mean percentage overshoot of scans starting cranial to T10 was $19.6 \%$, with a mean overshoot percentage of $8.6 \%$ for scans started at T10 and caudal to the level. Overshoot percentage is graphically presented in figure 2 demonstrating a reduction in overshoot percentage the more distal the CT KUB starts.

Table 1: Percentage CTKUB scan overshoot per vertebral level at which the scan was started.

\begin{tabular}{|l|l|}
\hline Scan start level & Average percentage overshoot \\
\hline T7 & 23.1 \\
\hline T8 & 17.9 \\
\hline T9 & 17.9 \\
\hline T10 & 12.7 \\
\hline T11 & 12.1 \\
\hline T12 & 9.1 \\
\hline L1 & 0.4 \\
\hline
\end{tabular}

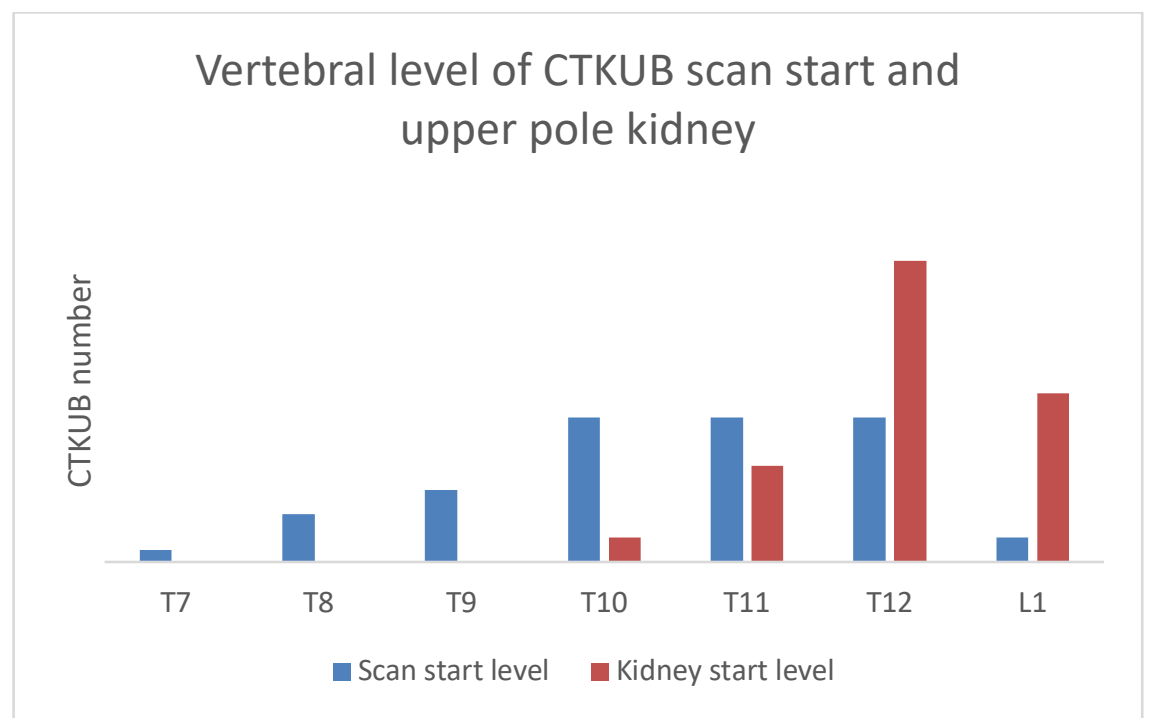

Figure 1: Number of CTKUB's performed at each vertebral level with the number patients whose kidneys upper pole commenced at that level. 
Number of CTKUB Vs Scan start level, \% overshoot and kidney level

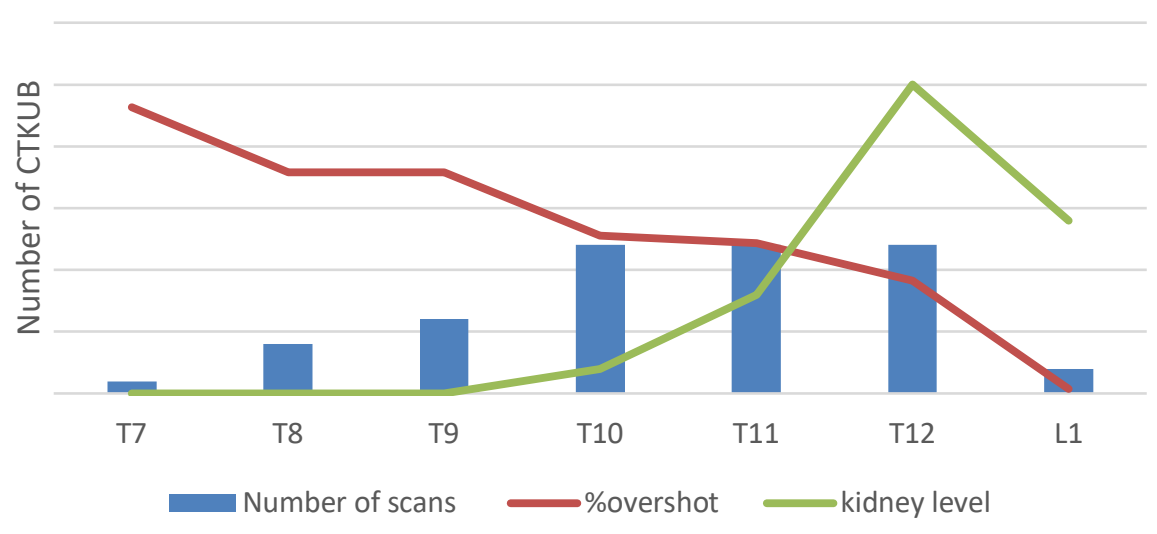

Figure 2: A graph depicting number of CT KUB scans which started at each level. Percentage overshoot and upper pole kidney lever are plotted over the graph to allow visualisation of overshoot relative to scan start location and anatomical kidney location.

\section{Discussion}

The aim of this retrospective audit was to investigate CTKUB scans performed at a teaching hospital with the view to assess percentage scanning overshoot and subsequently make recommendations on how exposure to ionising radiation can be reduced when performing CTKUB.

Lending to the characteristic symptoms of renal colic CTKUB is often performed to confirm diagnosis and formulate a management plan $^{3-5}$. Given the specific clinical question, the investigation must fully image the urinary tract however any tissue imaged cranial to the upper pole of the kidney is excess. The literature base suggests the use of the T10 level as a starting point for CTKUB imaging protocol ${ }^{15}$. This is suggested on the basis that T10 is cranial enough to capture the kidneys in the vast majority of individuals and to prevent under-scanning, were a portion of the urinary tract is not imaged proximal to the CTKUB start site ${ }^{15}$.

As can be seen in figures 1 and 2 a large number of scans are conducted at T9 or higher, with table 1 demonstrating that scans started above the level of T10 have the highest percentage overshoot and therefore the greatest delivery of unnecessary ionising radiation. This reinforces the literature suggested starting level of T10. Furthermore, this has been shown to translate to a reduction in ionising radiation. Previous studies which have implemented a T10 CTKUB starting protocol have achieved a mean ionising dose reduction to patients of $16-25 \% \%^{16-17}$. This is especially important given that renal stone disease often affects a young demographic and interval imaging is often required ${ }^{11,18}$.

Other literature has even suggested that although T10 allows for a degree of human error and anatomical variance in upper pole of kidney level, T11 would further reduce over-scanning in CTKUB and would not risk under scanning ${ }^{11}$. Uldin et al designed a protocol to scan starting at T11 and noted $0 \%$ underscanning and a $62.4 \%$ reduction in overall scanning length ${ }^{11}$. Similarly, Cavenagh et al suggested a T10 starting protocol however acknowledged that had they used T11 as a starting point none of their included participants would have been underscanned ${ }^{15}$. Our results identified three individuals (6\%) within the study were upper pole of kidney started at T10 hence a blanket T11 CTKUB starting protocol would have under-scanned these patients. Depending on the 
degree of under-scanning, repeat CT scanning may be required if vital anatomy is missed which would: increase exposure to ionising radiation; create a diagnostic delay for the patient and further strain CT scanner appointment slots. This suggests that although a T11 scanning protocol will be sufficient for the majority of patients and reduce radiation exposure, it is likely to under-scan a small percentage of patients undergoing CTKUB.

The results of this audit indicate that a CTKUB protocol which starts at the level of T10 would capture all of the necessary anatomy whilst minimising under-scanning risk. Given that $22 \%$ of scans started cranial to the level of T10, this protocol would minimise average percentage over scanning and radiation exposure. However, it must be noted that blanket protocol that allows for anatomical variance will always over-scan to a degree, this needs to be weighed up against risk of under-scanning and its subsequent detriment to the management of the patient.

Moving forward the study has met its objectives and highlights the need for implementation of a T10 CTKUB protocol. This protocol would need to be communicated to the radiography team and visual prompts in the form of posters detailing a protocol presented to maximise compliance. A potential limitation to adherence to protocol could be locum or temporary radiographers for the trust which may not be familiar with the protocol. Following a period of monitoring, the compliance to protocol would need to be re-audited. Further analysis can be collected comparing during office hours and out of office hours compliance to protocol as well as comparing permanent staff to temporary shift staff to help further identify areas which are least compliant.

\section{Conclusion}

In summary $22 \%$ of CTKUB scans started cranial to a T10 starting position. Implementation of a T10 start protocol will reduce the mean axial overshoot percentage from $19.6 \%$ to $8.6 \%$. This suggests implementing a T10 starting protocol at the teaching hospital which will have the effect of minimising ionising radiation exposure whilst preventing under-scanning.

\section{Acknowledgements}

The PACS radiology admin team for assisting with achieving an audit list.

\section{References}

1. Ziemba J, Matlaga B. Epidemiology and economics of nephrolithiasis. Investigative and Clinical Urology. 2017;58(5):299.

2. Leslie S, Sajjad H, Murphy P. Renal Calculi [Internet]. Ncbi.nlm.nih.gov. 2021 [cited 3 November 2021]. Available from: https://www.ncbi.nlm.nih.gov/books/NBK442 014/

3. Nephrolithiasis Clinical Presentation: History, Physical Examination, Complications [Internet]. Emedicine.medscape.com. 2021 [cited 3 November 2021]. Available from: https://emedicine.medscape.com/article/43709 6-clinical

4. I Refer Guidelines [Internet]. 2021 [cited 3 November 2021]. Available from: https://www.irefer.org.uk/guidelines

5. Overview | Renal and ureteric stones: assessment and management | Guidance | NICE [Internet]. Nice.org.uk. 2021 [cited 3 November 2021]. Available from: https://www.nice.org.uk/guidance/ng118

6. Fontarensky M, Alfidja A, Perignon R, Schoenig A, Perrier C, Mulliez A et al. Reduced Radiation Dose with Model-based Iterative Reconstruction versus Standard Dose with Adaptive Statistical Iterative Reconstruction in Abdominal $\mathrm{CT}$ for Diagnosis of Acute Renal Colic. Radiology. 2015;276(1):156-166.

7. Moore C, Daniels B, Singh D, Luty S, Gunabushanam G, Ghita M et al. Ureteral Stones: Implementation of a Reduced-Dose CT Protocol in Patients in the Emergency Department with Moderate to High Likelihood of Calculi on the Basis of STONE Score. Radiology. 2016;280(3):743-751.

8. Tenant S, Pang C, Dissanayake P, Vardhanabhuti V, Stuckey C, Gutteridge C et al. Intra-patient comparison of reduced-dose model-based iterative reconstruction with standard-dose adaptive statistical iterative 
reconstruction in the CT diagnosis and followup of urolithiasis. European Radiology. 2017;27(10):4163-4172.

9. Rob S, Bryant T, Wilson I, Somani B. Ultralow-dose, low-dose, and standard-dose CT of the kidney, ureters, and bladder: is there a difference? Results from a systematic review of the literature. Clinical Radiology. 2017;72(1):11-15.

10. Maguire J, Gray K. Computed tomography (CT) kidneys, ureters and bladder (KUB) how low can you go?. Clinical Radiology. 2015;70:S12.

11. Uldin H, McGlynn E, Cleasby M. Using the T11 vertebra to minimise the CT-KUB scan field. The British Journal of Radiology. 2020;93(1110):20190771.

12. Netke T, Spurr M, Vosough A. It is time to think inside the (collimation) box; a quality improvement project to reduce over-scanning in CT scanning of the kidneys, ureters and bladder. The British Journal of Radiology. 2020;93(1112):20200068.

13. Soriano RM, Penfold D, Leslie SW. Anatomy, Abdomen and Pelvis, Kidneys. [Updated 2021
Jul 26]. In: StatPearls [Internet]. Treasure Island (FL): StatPearls Publishing; 2021 Jan. Available from: https://www.ncbi.nlm.nih.gov/books/NBK482 385/

14. Fundamentals of Body CT. Third edition. Webb WR, Brant WE, Major NM. Elsevier Health Sciences Division. November 2005. Page 276.

15. Maguire J, Gray K. Computed tomography (CT) kidneys, ureters and bladder (KUB) how low can you go?. Clinical Radiology. 2015;70:S12.

16. McCollough C, Chen G, Kalender W, Leng S, Samei E, Taguchi K et al. Achieving Routine Submillisievert CT Scanning: Report from the Summit on Management of Radiation Dose in CT. Radiology. 2012;264(2):567-580.

17. Sung M. Current status of low dose multidetector CT in the urinary tract. World Journal of Radiology. 2011;3(11):256.

18. Romero V, Akpinar H, Assimos DG. Kidney stones: a global picture of prevalence, incidence, and associated risk factors. Rev Urol. 2010;12(2-3):e86-e96. 